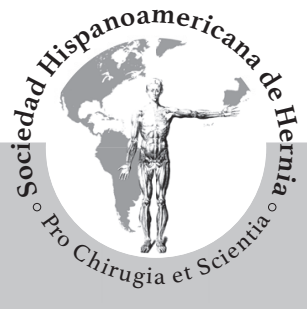

Nota clínica

\title{
Hernia inguinal incarcerada por un fecaloma asociado a un dolicomegacolon idiopático
}

\section{Incarcerated inguinal hernia due to a fecalloma associated with idiopathic dolicomegacolon}

\section{Francisco Javier Ortiz de Solórzano Aurusa ${ }^{1}$, Zahira Gómez Carmona ${ }^{1}$, Carlos Ferreras García', Elvira González Obeso ${ }^{2}$, Beatriz de Andrés Asenjo', Juan Beltrán de Heredia Rentería'}

\section{'Servicio de Cirugía General y Aparato Digestivo. Hospital Clínico Universitario de Valladolid. ${ }^{2}$ Servicio de Anatomía Patológica.} Hospital Clínico Universitario de Valladolid.

Recibido: 30/11/2016

Aceptado: 14/01/2017

\section{Palabras clave:}

Incarceración. Hernia inguinal.

Fecaloma. Dolicomegacolon

\section{Resumen}

Presentamos el caso clínico de un paciente con antecedentes de estreñimiento de larga evolución. Presentaba una hernia inguinal derecha incarcerada, secundaria a la presencia de un fecaloma gigante asociado a un dolicomegacolon idiopático. Se intervino de forma programada realizando una hernioplastia inguinal y una colectomía sigmoidea . La evolución posoperatoria fue satisfactoria. Se realiza una revisión de esta rara entidad de la que no hemos encontrado ninguna referencia bibliográfica.
Key words:

Incarceration. Inguinal hernia. Fecaloma. Dolichomegacolon

\section{Abstract}

We present the case of a patient with a long-standing constipation history. He had an incarcerated inguinal hernia, secondary to an giant fecaloma associated to an idiopathic dolichomegacolon. We realized a non urgent surgery, performing an inguinal hernioplasty and a sigmoid colectomy. The postopeative course was satisfactory. A review of this rare entity of which have not found bibliographic references is done.

* Autor para correspondencia. F. J. Ortiz de Solórzano Aurusa. Servicio de Cirugía General y Aparato Digestivo. Hospital Clínico Universitario de Valladolid. C/ Ramón y Cajal s/n 47005 Valladolid (España)

Correo electrónico: fjortiz@saludcastillayleon.es

2255-2677/C 2017 Sociedad Hispanoamericana de Hernia. Publicado por Arán Ediciones, S.L. Todos los derechos reservados

http://dx.doi.org/10.20960/rhh.39 


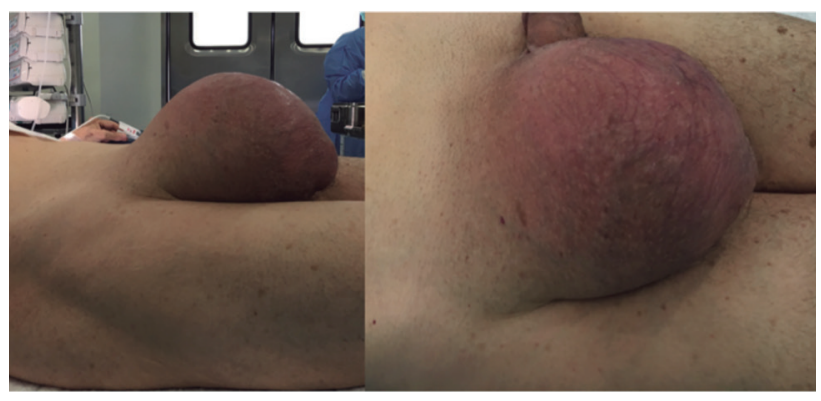

Figura 1. Hernia inguinoescrotal derecha incarcerada.Visión lateral y anteroposterior.

\section{Introducción}

El estreñimiento es un padecimiento crónico que afecta a numerosas personas. En los ancianos se considera un problema sanitario que genera muchas consultas y gasto farmacológico (1). En los casos más severos, el estreñimiento puede dar lugar a la formación de un fecaloma, que se define como una masa de heces duras localizada generalmente en el recto o en el colon sigmoides.

La incarceración herniaria de un fecaloma es una situación excepcional: en la bibliografía revisada solamente hay publicados dos casos similares, ambos en niños de corta edad (2,3). El paciente que presentamos sería el primer caso de incarceración herniaria por un fecaloma en el adulto, motivo de esta publicación.

\section{Caso clínico}

Varón de 75 años, estudiado en el Servicio de Aparato Digestivo por un estreñimiento de larga evolución, diagnosticado de dolicomegacolon, fecaloma sigmoideo y síndrome de Chilaiditi. Se descartó enfermedad de Hirschsprung por biopsia rectal. La serología frente al Tripanosoma cruzzi fue negativa (el paciente había vivido durante unos años en Brasil). Otros antecedentes que presentaba eran asma intrínseca, flutter auricular tratado con ablación de istmo cavotricuspídeo y una intervención por vía endoscópica de una hipertrofia prostática benigna y litiasis vesical. En tratamiento habitual con laxantes por vía oral y enemas.

Remitido a consultas externas del Servicio Cirugía por presentar una hernia inguinal derecha de dos años de evolución. En la exploración física, en la región inguinal derecha se apreciaba una gran tumoración de consistencia pétrea, no dolorosa e irreductible, compatible con hernia inguinal incarcerada, sin poder descartar una lesión de origen tumoral (fig. 1).

Se solicitó una tomografía computarizada en la que se confirmó la existencia de una hernia inguinal derecha, en cuyo saco herniario se encontraba el colon sigmoideo con un fecaloma gigante en su interior. El resto del colon estaba dilatado y ocupado por material fecaloideo (fig. 2).

El estudio preoperatorio no presentaba alteraciones, por lo que se le intervino quirúrgicamente de forma programada bajo anestesia general. Se realizó una incisión oblicua por encima del pliegue inguinal derecho; tras la apertura del conducto inguinal se apreció una gran hernia directa, con un saco herniario que contenía el colon sigmoideo dilatado con el fecaloma en su interior. Se le practicó una colectomía sigmoidea por vía inguinal, pero al apreciar que el colon distal estaba dilatado y que su abordaje y exéresis completa por vía inguinal era impracticable, se le realizó una laparotomía media infraumbilical para ampliar la resección al recto superior. Para la reparación de la hernia se realizó una hernioplastia según la técnica descrita por Celdrán et al. (4) (fig. 3), con una malla de polipropileno macroporosa de baja densidad de $15 \times 15 \mathrm{~cm}$ (Dipromed ${ }^{\circledR}$, Italia). La reconstrucción del tránsito digestivo se realizó con una anastomosis colorrectal término-terminal con sutura mecánica EEA ${ }^{\mathrm{TM}}$ núm. 31 (Covidien-Medtronic ${ }^{\circledR}$, Mineápolis, EE. UU.). El posoperatorio cursó sin incidencias, y recibió el alta hospitalaria a los 6 días de la intervención.

La pieza quirúrgica tenía una longitud de $40 \mathrm{~cm}$ y presentaba un diámetro que oscilaba entre $6 \mathrm{~cm}$ en el extremo proximal y $20 \mathrm{~cm}$ en el distal. La superficie serosa de la pieza era lisa y brillante, con ligera congestión vascular. En la apertura de la pieza se observó

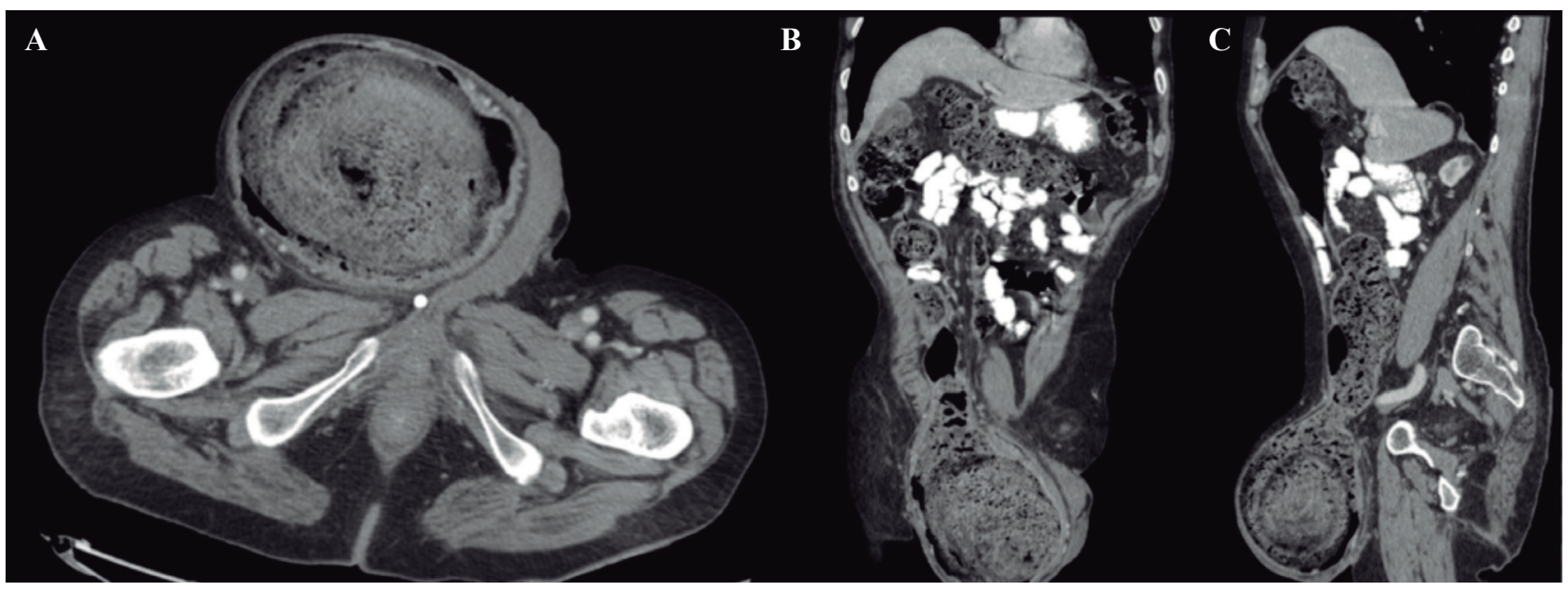

Figura 2. Tomografía computarizada en la que se aprecia un fecaloma gigante en colon sigmoideo causando incarceración de una hernia inguinal derecha. A. axial; B. Coronal; C. Lateral 


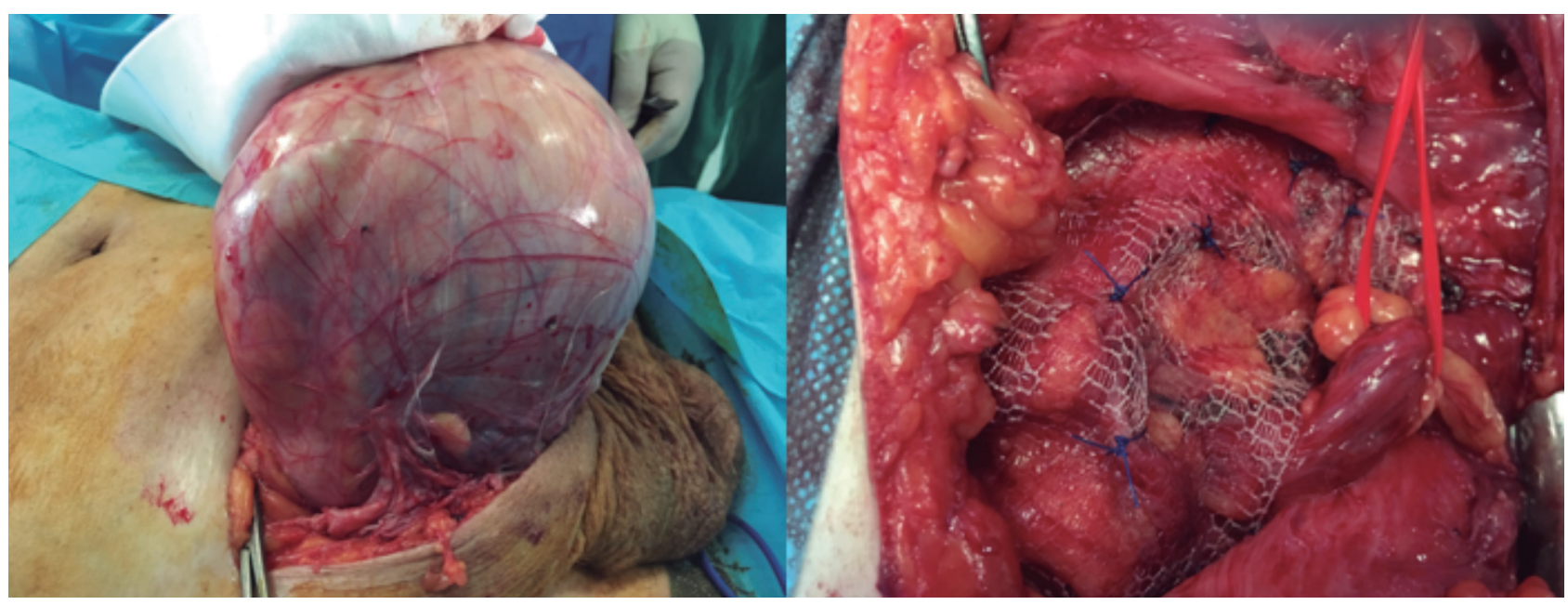

Figura 3. Aspectos intraoperatorios, gran saco herniario con el colon sigmoides, hernioplastia con malla de polipropileno macroporosa de baja densidad.

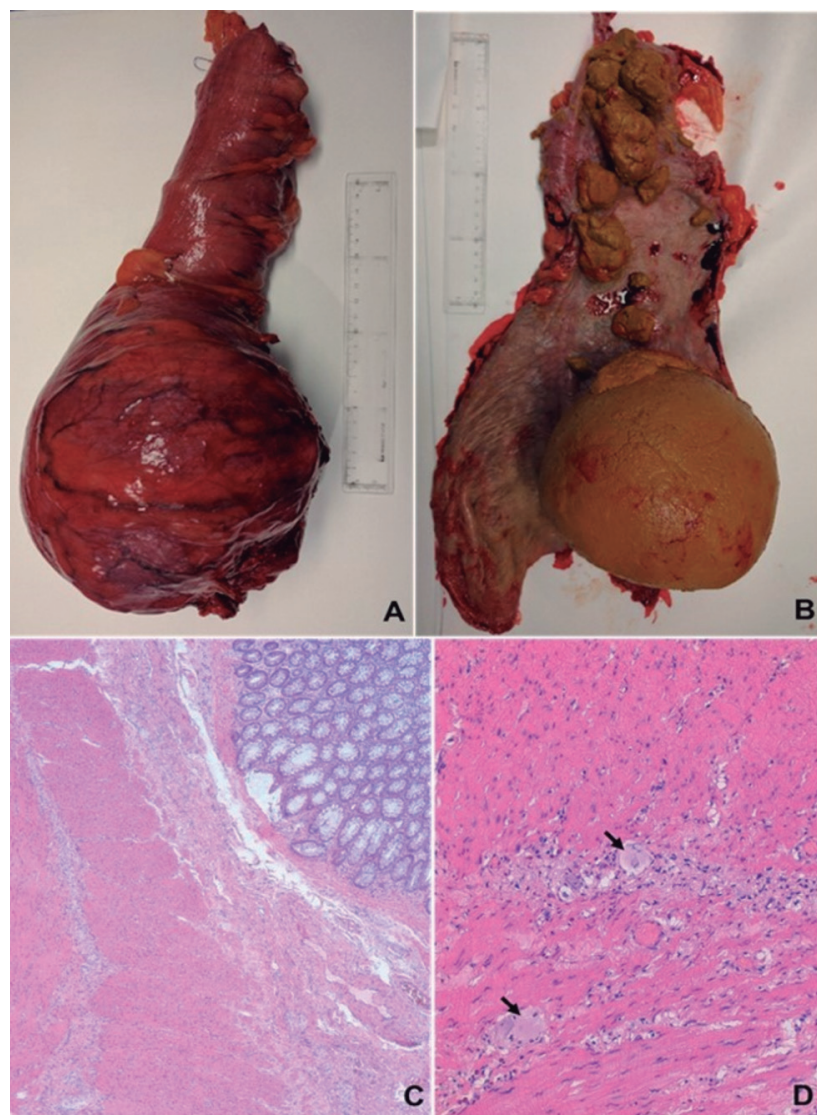

Figura 4. A. Pieza de resección de recto sigma con extremo distal marcadamente dilatado. B. A la apertura se objetiva un gran fecaloma de $18 \mathrm{~cm}$ de diámetro y marcado adelgazamiento de la pared del intestino grueso. C. Sección transversal de la pared en la que se objetiva el adelgazamiento y la prominencia de los plexos submucoso y mientérico. (Hematoxilina-Eosina, 40x). D. Detalle de un plexo mientérico donde se marcan con flechas dos células gangliónicas. Se objetiva la hiperplasia e hipertrofia de los haces nerviosos del plexo. un gran fecaloma de $18 \mathrm{~cm}$ de diámetro y consistencia dura que ocupaba la luz. El extremo proximal de la pieza presentaba contenido intestinal de parecidas características, pero de consistencia más blanda. La pared del colon estaba adelgazada y su espesor oscilaba desde los 4-5 mm en el extremo proximal hasta los 2-3 $\mathrm{mm}$ en la zona más dilatada. Macroscópicamente no se observaron alteraciones en la mucosa. Histológicamente se observaron células ganglionares normales en los plexos submucoso y mientérico en todos los tramos de la pieza. Existía un marcado adelgazamiento de todas las capas de la pared intestinal y de ligera a moderada hiperplasia e hipertrofia de los plexos nerviosos submucoso y mientérico (fig. 4).

Revisado en consultas externas, a los dos y cuatro meses de la intervención el paciente refirió un ritmo de deposición normal, sin necesidad de laxantes, y presentaba un buen estado de las heridas operatorias.

\section{Discusión}

Los fecalomas se presentan generalmente en pacientes con historia de un estreñimiento crónico. Sus causas más frecuentes son enfermedad de Hirschsprung, enfermedad de Chagas (Tripanosoma cruzzi), lesiones de la médula espinal y pacientes con enfermedades psiquiátricas, malformaciones anorrectales, enfermedades inflamatorias o neoplásicas del colon, etc. $(5,6)$.

Los fecalomas pueden ser el origen de masas abdominales $(7,8)$ y la causa de complicaciones urológicas por compresión de estructuras anatómicas (9). También se han descrito oclusiones intestinales o perforaciones estercoráceas, en ocasiones con un desenlace fatal $(10,11,12)$.

El tratamiento de los fecalomas es generalmente médico, con laxantes, enemas, fragmentación y extracción digital directa o bajo sedación. A veces ha sido efectiva la extracción endoscópica (13), y raramente se requiere una laparotomía (12).

La herniación de un fecaloma es un hecho excepcional. Los dos casos registrados previamente en la bibliografía se diferen- 
cian del nuestro por tratarse de impactaciones fecales, es decir, un conglomerado de heces debido a la coproestasis con una consistencia más blanda que los fecalomas. Se presentaron en niños, en la región inguinal izquierda, sin enfermedad previa en el colon, y se resolvieron con una herniorrafia tras la movilización de las heces. Nuestro caso tiene las singularidades de presentarse en edad adulta, la de localizarse en la región inguinal derecha por herniación de un dolicocolon con un fecaloma en su interior y la imposibilidad de su reducción sin colotomía o colectomía.

La táctica quirúrgica inicial fue la de realizar la intervención por vía inguinal, pero ante los hallazgos intraoperatorios se asoció una laparotomía media infraumbilical para ampliar la resección al rectosigma. Para la realización de la hernioplastia se optó por la técnica descrita por Celdrán et al. (4), con fijación de la prótesis al ligamento de Cooper, ya que se refuerza la región inguinal cubriendo los orificios directos, indirectos y crural. La malla empleada fue de polipropileno macroporosa de baja densidad, pues al ser una cirugía contaminada y potencialmente sucia, este material soportaría mejor una posible infección posoperatoria.

La etiología del megacolon y del megarrecto idiopático es desconocida. Se ha planteado que puedan ser causados por una anormalidad funcional nerviosa asociada a un engrosamiento del músculo liso, sin alteraciones en la arquitectura de la inervación (14). Los hallazgos histológicos encontrados en los pacientes intervenidos por un fecaloma son los siguientes: disminución de células ganglionares e intersticiales de Cajal asociadas a un engrosamiento de las capas musculares (10), hipertrofia de fibras nerviosas con ausencia de células ganglionares en el plexo mientérico de Auerbach (5) y una atrofia de la mucosa y submucosa (8). En nuestro paciente, las alteraciones morfológicas observadas fueron un marcado adelgazamiento de la pared del intestino con hipertrofia e hiperplasia de los plexos submucoso y mientérico que, en nuestra opinión, son la consecuencia de un cuerpo extraño (en este caso un fecaloma) impactado durante largo tiempo.

Como conclusiones del caso presentado, creemos necesario realizar un correcto estudio y tratamiento de los pacientes con estreñimiento crónico y, sobre todo, en los casos asociados a un fecaloma, para prevenir sus complicaciones (que pueden ser potencialmente graves).

\section{Bibliografía}

1. Linberg G, Hamid S, Malfertheiner P, Thomsen O, Bustos L, Garissch J, et al. World Gastroenterology Organisation Global Guideline. Constipation- A global Perspective. J Clin Gastroenterol 2011;45:483-487.

2. Roy AK, Ghildiyal JP. Impaction of feces in a loop of sigmoid colon: A rare cause of incarceration of inguinal hernia in children. International J Surg. 2008;6:e7-e8.

3. Medina-Andrade MA, Gallardo-Meza AF, Piña-Garay MA, González-Sánchez JM. Hernia inguinal encarcelada por impacto fecal de sigmoides en un lactante. Acta Pediatr Mex. 2010;31:29-31.

4. Celdrán A, Vorwald P, Meroño E, García-Ureña MÁ. A single technique for polypropilene mesh hernioplasty of inguinal and femoral hernias. Surg Gynecol Obstet. 1992;175:359-261.

5. Garisto JD, Campillo L, Edwards E, Harbour M, Ermocilla R. Giant fecaloma in a 12-year-old-boy: a case report. Cases Journal. 2009;127-130.

6. Altomare D, Rinaldi M, Sallustio PL, Armenise N. Giant fecaloma in a adult with severe anal stricture caused by anal imperforation treated by proctocolectomy and ileostomy: Report of a case. Dis Colon Rectum. 2009;52:534-537.

7. Caiazzo P, De Martino C; Del Vecchio G, Di Lascio P, Marasco M, Laviani F, et al. Megacolon for a giant fecaloma with unluky outcome. Case report anr review of the literature. Ann Ital Chir. 2013;84:319-322.

8. Yucel AF, Akdogan RA, Gucer H. A giant abdominal mass: Fecaloma. Clin Gastroenterol Hepatol. 2012;10:e9-e10.

9. Park JS, Park TJ, Hwa JS, Seo JH, Park ChH, Youn PH. Acute urinary retention in a 47-month-old gril caused by the giant fecaloma. Pediatr Gastroenterol Hepatol Nutr. 2013;16:200-2005.

10. Ribas Y, Bargalló J, Lamas S, Aguilar F. Idiopathic sigmoid megacolon with fecal impactation and giant calcified fecaloma. Am Surg. 79;2:e96-e97.

11. Mushtaq M, Shah MA, Malik AA, Wani KA, Takur N, Parray FQ. Giant fecaloma causing small bowel obstruction: Case report and review of the literatura. Bull Emerg Trauma. 2015;3:70-72.

12. Rajagopal A, Martin J. Giant fecaloma with idiopathic sigmoid megacolon. Report of a case and review of the literatura. Dis Colon Rectum. 2002;45:833-835.

13. Sakai E, Inokuchi Y, Inamori M, Uchiyama T, Lida H, Takahasi H, et al. Rectal fecaloma: Successful treatment using endoscopic removal. Digestion. 2007;75:198.

14. Gatusso JM, Kamm MA, Talbot IC. Pathology of idiopathic megarectum and megacolon. Gut. 1977;41:252-257. 